\title{
Acanthocéphales des esturgeons de la mer Caspienne
}

\author{
par Yves J. GOLVAN et Baba MOKHAYER \\ Laboratoire de Parasitologie et Pathologie parasitaire \\ Faculté de Médecine de Paris-St-Antoine, 27, rue de Chaligny, F 75012 Paris \\ et Laboratoire d'Ichthyologie, Faculté vétérinaire, Téhéran, Iran
}

\begin{abstract}
Résumé
Description d'une nouvelle espèce d'Acanthocéphale chez les esturgeons de la Caspienne, Corynosoma caspicum n. sp.

\section{Summary}

\section{Sturgeons Acanthocephala from Caspian sea.}

Description of a new Acanthocephala, Corynosoma caspicum $\mathrm{n}$, sp., a parasite of the gut of three species of sturgeons on the iranian coast of the Caspian sea.
\end{abstract}

L'un de nous, dans le cadre du travail préparatoire à sa thèse de Doctorat es-Sciences Naturelles, a récolté dans l'intestin de divers esturgeons capturés sur le littoral iranien de la mer Caspienne, deux espèces d'Acanthocéphales.

Leptorhynchoides plagicephalus (Westrumb 1821) Kostylew 1924 (Echinorhynchus plagicephalus : Westrumb $1821=$ Echinorhynchus husonis Rudolphi $1819=$ Echinorhynchus acipenseris rutheni Rudolphi 1819).

Dans nos rézoltes, les hôtes de cette espèce sont Acipenser stellatus Pallas et Acipenser guldenstaedti Brandt. Plusieurs dizaines d'individus sexuellement mûrs ont été récoltés dans l'intestin de ces esturgeons. Nous avons constaté que l'anatomie si minutieusement décrite par Kostylew (1924) et les mensurations qu'il indique correspondent parfaitement aux exemplaires dont nous disposons.

Nous ne donnerons donc ici que quelques figures originales qui pourront venir compléter celles publiées par Kostylew dans son article princeps. 


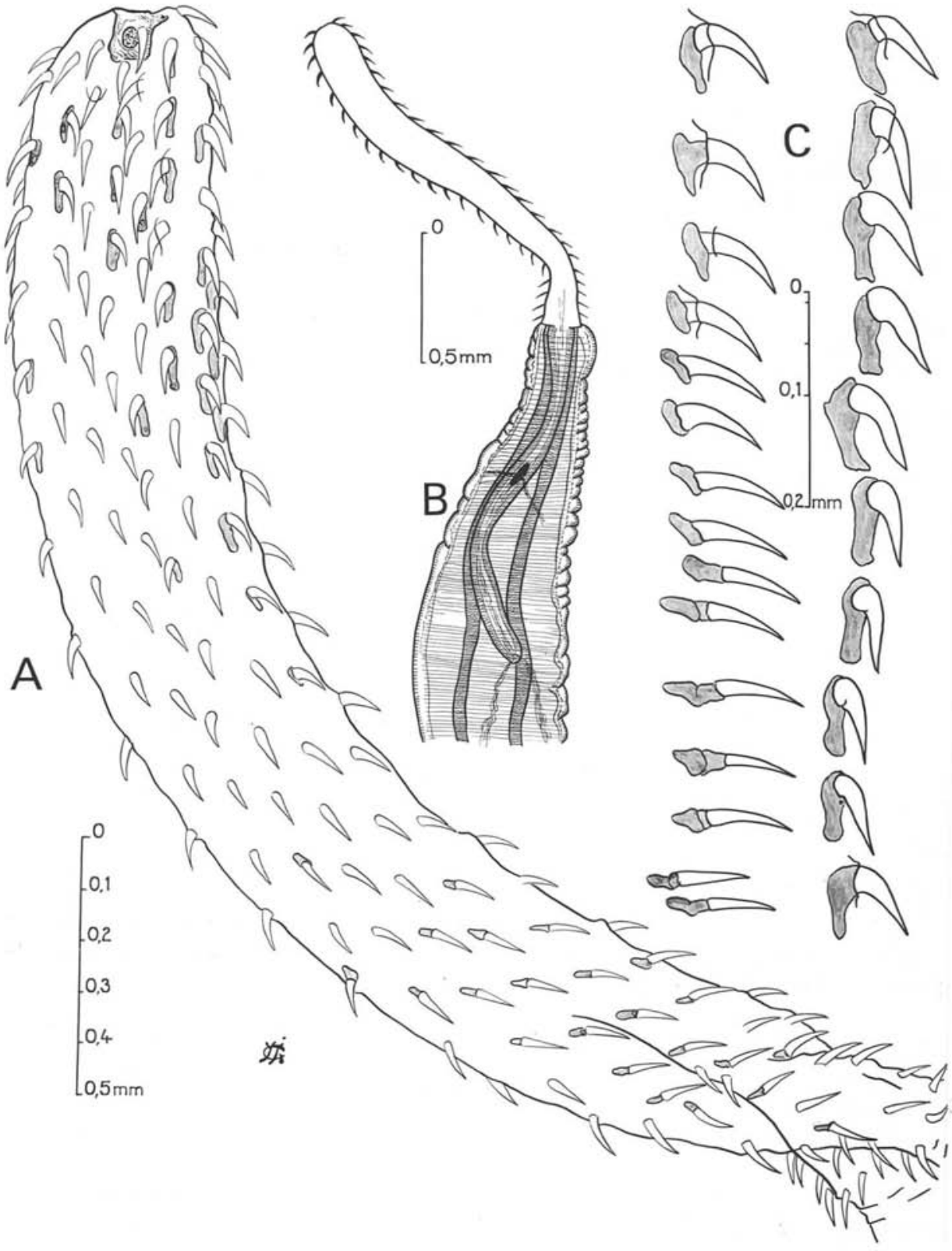

FIGURE 1. - Leptorhynchoides plagicephalus (Westrumb) Kostylew : A) Rostre d'un individu mâle; B) Partie antérieure d'un individu mâle; C) File de crochets du rostre, la partie antérieure étant à droite et la partie postérieure à gauche. 


\section{Corynosoma caspicum n. sp.}

Nous disposons de plusieurs centaines d'exemplaires de cette espèce provenant de l'intestin d'esturgeons capturés dans huit stations de pêche du littoral iranien de la Caspienne dans le secteur de Bendar Pahlevi au printemps et à l'automne 1971. Les poissons parasités appartiennent aux espèces Acipenser stellatus Pallas (5 individus positifs sur 72 examinés), Acipenser guldenstaedti Brandt (3 positifs sur 95 examinés) et Huso huso (Linné 1758) (1 positif sur 4 examinés). Aucune femelle de notre Acanthocéphale de contient d'œuf mûr.

Il s'agit d'un Acanthocéphale de petite taille. Les mâles ont, en moyenne, 3,925 $\mathrm{mm}$ de long et les femelles $4,675 \mathrm{~mm}$. La forme générale est fuselée avec une partie très renflée qui correspond approximativement au tiers antérieur du tronc. Le proboscis s'insère plus près de la face ventrale que de la face dorsale et, de plus, son grand axe est presque toujours incliné ventralement par rapport au grand axe du tronc. Le rostre est en fuseau bref. Il mesure $0,575 \mathrm{~mm}$ de long sur $0,260 \mathrm{~mm}$ de diamètre maximum chez les mâles contre $0,725 \mathrm{~mm}$ de long sur 0,270 à $0,320 \mathrm{~mm}$ de diamètre chez les femelles. Il est armé de 16 (plus rarement 18) files longitudinales de 10 crochets chacune. Les crochets supérieurs sont fins et possèdent des racines de forme simple à direction postérieure. Le cinquième crochet est brusquement beaucoup plus gros que les précédents $(0,043 \mathrm{~mm}$ de long sur $0,018 \mathrm{~mm}$ de large chez les mâles pour $0,060 \mathrm{~mm}$ sur $0,021 \mathrm{~mm}$ chez les femelles). C'est à hauteur de cette couronne de gros crochets que le diamètre du rostre est le plus grand. Les racines des grands crochets sont de forme simple alors que les racines des crochets nettement plus petits placés immédiatement en arrière (crochets VI et VII) sont en forme d'étoile à trois branches. Elles comprennent une apophyse supérieure directement dirigée vers l'apex du rostre et deux apophyses inférieures divergeant selon un angle d'environ $30^{\circ}$. Le crochet VII mesure $0,030 \mathrm{~mm}$ de long sur $0,013 \mathrm{~mm}$ de large chez les mâles et $0,048 \mathrm{~mm}$ de long sur $0,009 \mathrm{~mm}$ de large chez les femelles. Les 3 ou 4 derniers crochets de chaque file sont, en réalité, des épines à racines rudimentaires. Les lames de ces épines mesurent en moyenne 0,022 à $0,031 \mathrm{~mm}$ de long. Le cou est court, tronc-conique, long de 0,22 $\mathrm{mm}$ chez les mâles et de $0.475 \mathrm{~mm}$ chez les femelles.

La partie antérieure renflée du tronc mesure de 1 à $1,8 \mathrm{~mm}$ de long pour un diamètre de 0,91 à $1,30 \mathrm{~mm}$. Elle est recouverte d'un champ d'épines cuticulaires couvrant pratiquement toute la portion dilatée sur la face dorsale et se terminant en pointe inférieure sur la partie toute antérieure de la face ventrale de la partie postérieure cylindrique du tronc. Les épines cuticulaires les plus grosses sont situées dans la partie moyenne de la hauteur de ce champ et atteignent $0,055 \mathrm{~mm}$ de longueur totale. La partie postérieure du tronc est cylindrique, bien plus étroite et inerme. $\mathrm{Ce}$ segment est long de 1,45 à $1,75 \mathrm{~mm}$ pour un diamètre variant de 0,465 à $0,630 \mathrm{~mm}$. Les orifices génitaux sont entourés d'épines dont le nombre est d'environ 40 chez les mâles et d'environ 20 chez les femelles. Ces épines sont bien plus petites que les épines du champ antérieur. La partie postérieure du tronc est souvent invaginée dans l'un et l'autre sexes formant un vestibule génital. 


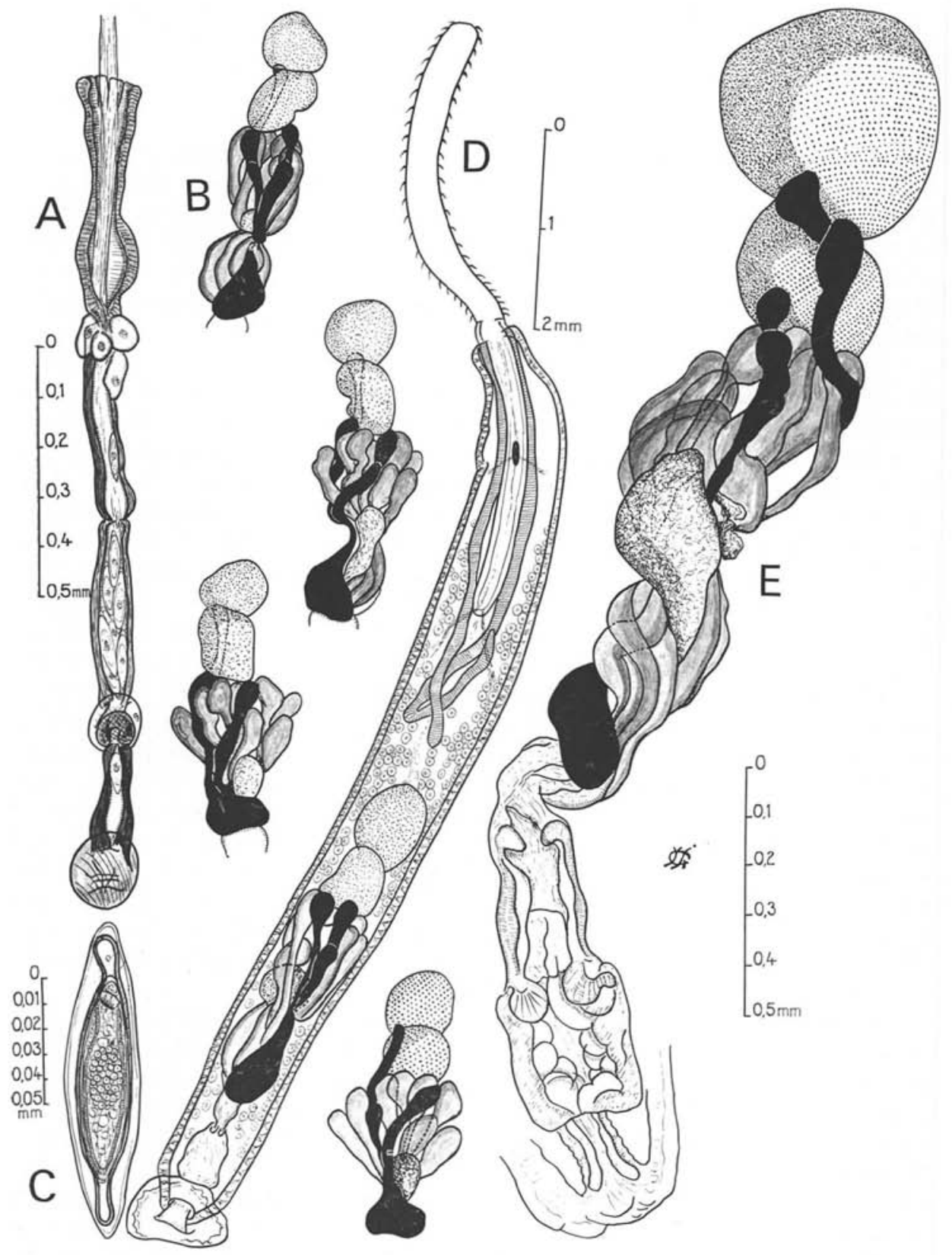

FIGURE 2. - Leptorhynchoides plagicephalus (Westrumb) Kostylew: A) Appareil génital femelle ; B) Appareil génital mâle à divers stades d'écrasement de la préparation. Noter les dilatations successives de canaux déférents et la grosse vésicule séminale cordiforme; C) Embryophore mûr ; D) Individu mâle in toto; E) Appareil génital mâle. 


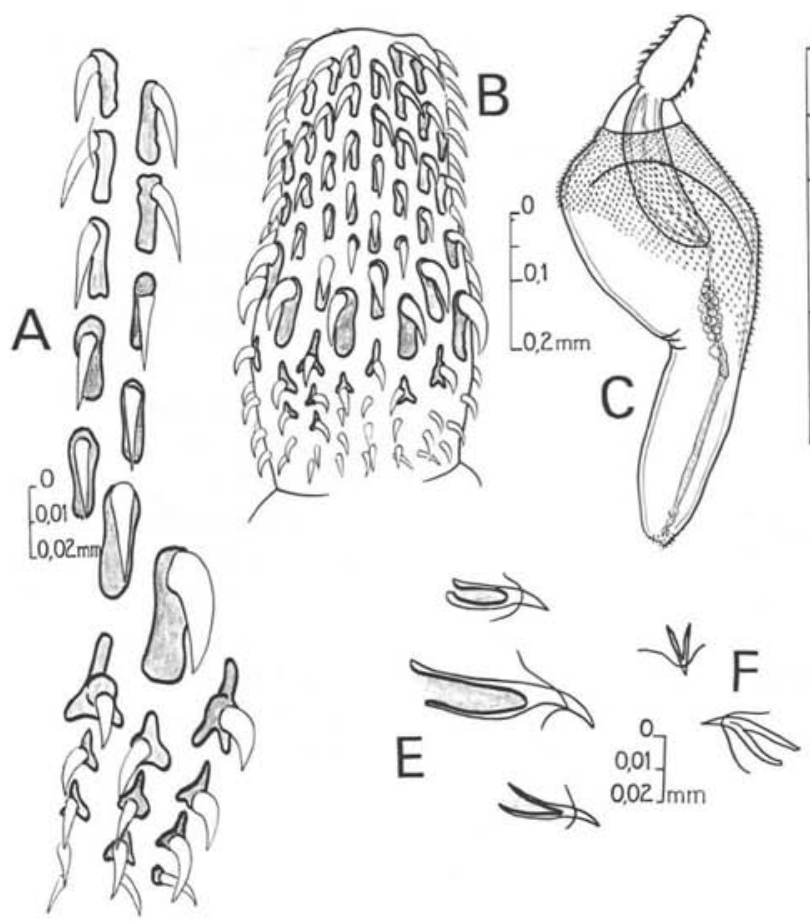

FIGURE 3. - Corynosoma caspicum n. sp.: A) Files de crochets du rostre; B) Rostre d'un individu mâle ; C) Femelle in toto; D) Appareil vaginal ; E) Epines cuticulaires antérieures. La plus grosse est une de celles situées à la partie moyenne de la partie ventrale du champ spinulé; F) Epines génitales; G) Mâle in toto.

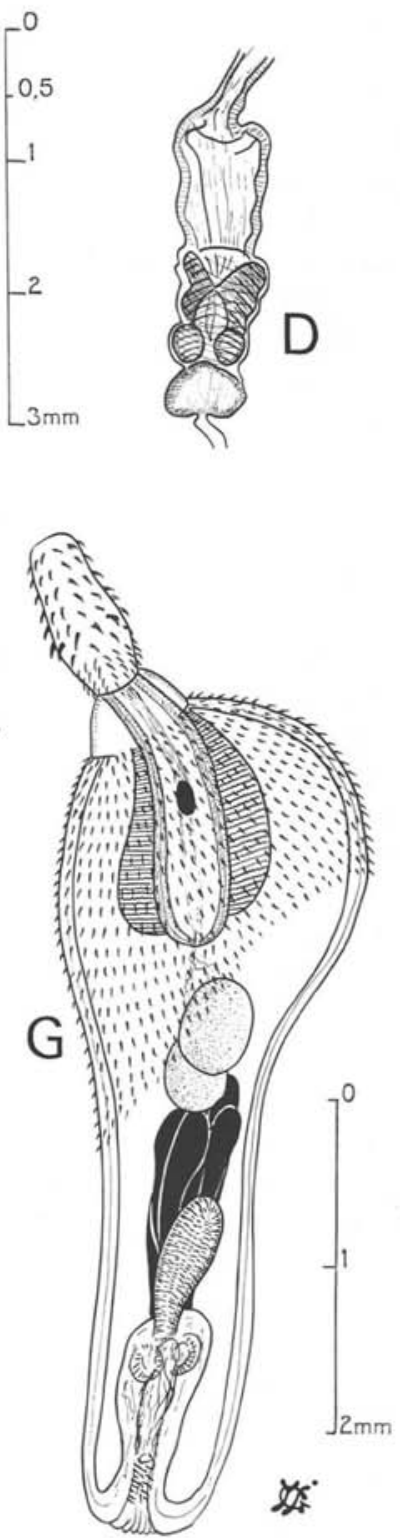


Le réceptacle du proboscis a une double paroi, il s'insère à la base du rostre et mesure environ 0,9 à $1,3 \mathrm{~mm}$ de long. Le ganglion cérébrö̈de est placé dans sa partie moyenne. Les lemnisques sont aplatis et un peu plus courts que le réceptacle. L'appareil génital mâle comprend deux testicules sphéroïdaux, d'environ $0,3 \mathrm{~mm}$ de long sur $0,25 \mathrm{~mm}$ de large, se recouvrant partiellement l'un l'autre. Il y a quatre glandes cémentaires en massue allongée à grosse extrémité supérieure. L'organe de Saefftigen est volumineux.

L'appareil génital femelle mesure $1,750 \mathrm{~mm}$ de long. L'entonnoir vaginal est bien développé. Le sphincter vaginal supérieur est nettement plus fort que le sphincter inférieur. Chez toutes nos femelles l'ovaire est en voie de fragmentation et cache la cloche utérine.

Nous considérons cette espèce comme nouvelle. En effet, jusqu'à ce jour, une seule espèce du genre Corynosoma a été signalée dans la Caspienne. Il s'agit de Corynosoma strumosum identifié par Dogiel et Bykhowski (1939) chez Phoca caspica sur le littoral soviétique. Nous pensons cependant que cette détermination ou cette assimilation mérite d'être confirmée car il n'est pas certain que le Phoque de la Caspienne héberge le même parasite que ses cousins de l'Océan arctique. Si l'on s'en tient à la détermination des auteurs russes, l'espèce que nous avons trouvée chez les esturgeons est nettement différente de $C$. strumosum. Ce dernier est en effet nettement plus grand (jusqu'à $9 \mathrm{~mm}$ de long), il possède de 22 à 24 files de crochets dont 8 à 10 crochets vrais suivis de 5 à 6 épines basales. Le dimorphisme sexuel est peu apparent alors qu'il est très net chez notre espèce. De plus la spinulation du tronc est, chez $C$. strumosum, en continuité avec la spinulation génitale alors que chez nos exemplaires des deux sexes, le champ antérieur est nettement séparé du champ génital par une longue zone inerme occupant presque toute la face ventrale de la portion cylindrique du tronc. Il faut enfin signaler la morphologie très particulière des racines des crochets des couronnes VI et VII.

Nous proposons pour cette espèce le nom de Corynosoma caspicum n. $\mathrm{sp}$.

\section{Bibliographie}

Dogiel (V.-A.) et BYKHowsKI (B.-E.), 1938. - Parasitologie de la mer Caspienne (en russe). Akad. Nauk. Léningrad, VII.

Kostylew (N.-N.), 1924. - Le genre Leptorhynchoides, nouveau genre d'Acanthocéphale parasite de Poissons. Ann. Parasit. hum. comp., 11 (3), 214-223.

RUDOLPHI (C. A.), 1819. - Entozoorum Synopsis cui accedunt mantissa duplex et indices locupletissimi, A. Rücker, édit., Berolini, I-X + 1-811 (pl. I-III), 63-81-309-336572-600-665-674.

Westrumb (A. H. L.), 1821. - De Helminthibus Acanthocephalis. Commentatio historicoanatomica adnexo recensu animalium, in: Museo Vindobonensis circa helminthes dissectorum et singularum speciarum harum in illis repertarum, Hannoverae Helwing, édit. 